\title{
Operando XAS Study of Atomic Phase Reversibility with Wavelet Transform in the Lithium-rich Manganese Based Oxide Cathode
}

Taehoon Kim, ${ }^{*, t, \omega}$ Bohang Song, ${ }^{\dagger}$ Alexander J.G. Lunt, ${ }^{\dagger}$ Giannantonio Cibin, ${ }^{\ddagger}$ Andrew J. Dent, ${ }^{\ddagger}$ Li Lu, ${ }^{\S}$ and Alexander M. Korsunsky

Supplementary Table 1 | EXAFS fitting parameters at $\mathrm{Mn}$ K-edge on the graphene incorporated $\mathrm{Li}\left(\mathrm{Li}_{0.2} \mathrm{Mn}_{0.54} \mathrm{Ni}_{0.13} \mathrm{CO}_{0.13}\right) \mathrm{O}_{2}$. $\mathrm{CN}$ is the coordination number, $\mathrm{R}$-range is the fitting range, and $\mathrm{TM}$ is the transition metals ( $\mathrm{Mn}, \mathrm{Co}$, and $\mathrm{Ni}$ ). R-factor characterizes misfits between the experimental and theoretical data during EXAFS modelling. Amplitude reduction factor $\left(S_{0}{ }^{2}\right)$ was 0.7. $\sigma^{2}$ Indicates the 'Mean Squared Relative Displacement' between the absorber and scatterer.

\begin{tabular}{ccccccccc}
\hline $\begin{array}{c}\text { Charge } \\
\text { point }\end{array}$ & $\begin{array}{c}\text { Chemical } \\
\text { Potential }(V)\end{array}$ & R-range $(\AA)$ & $\mathrm{CN}$ & $\begin{array}{c}\mathbf{1}^{\text {st }} \text { shell } \\
\text { Mn-O } \\
(\AA)\end{array}$ & $\begin{array}{c}\mathbf{2}^{\text {nd }} \text { shell } \\
\text { Mn-TM } \\
(\AA)\end{array}$ & $\begin{array}{c}\mathbf{1}^{\text {st }} \text { shell } \\
\left(\sigma^{2}\right)\end{array}$ & $\begin{array}{c}2^{\text {nd }} \text { shell } \\
\left(\sigma^{2}\right)\end{array}$ & R-factor \\
\hline REF. & 3.6098 & $1-3.6$ & 6 & 1.91074 & 2.80974 & 0.00355 & 0.00813 & 0.0134557 \\
1 & 4.326 & $1-3.65$ & 6 & 1.90484 & 2.80384 & 0.00311 & 0.0089 & 0.0101629 \\
2 & 4.5064 & $1.07-3.55$ & 6 & 1.90789 & 2.80689 & 0.00331 & 0.01072 & 0.0085745 \\
3 & 4.7507 & $1-3.65$ & 6 & 1.90497 & 2.78477 & 0.00347 & 0.00596 & 0.0099921 \\
4 & 3.8513 & $1-3.55$ & 6 & 1.90648 & 2.80548 & 0.00294 & 0.00826 & 0.0097994 \\
5 & 2.9265 & $1-3.55$ & 6 & 1.91047 & 2.80947 & 0.00318 & 0.00751 & 0.013805 \\
6 & 2.1822 & $1-3.5$ & 6 & 1.91096 & 2.79076 & 0.00401 & 0.00938 & 0.0163302
\end{tabular}

Supplementary Table 2 | EXAFS fitting parameters at Co K-edge on the graphene incorporated $\mathrm{Li}\left(\mathrm{Li}_{0.2} \mathrm{Mn}_{0.54} \mathrm{Ni}_{0.13} \mathrm{Co}_{0.13}\right) \mathrm{O}_{2}$. $\mathrm{CN}$ is the coordination number, $\mathrm{R}$-range is the fitting range, and $\mathrm{TM}$ is the transition metals (Mn, Co, and $\mathrm{Ni}$ ). R-factor characterizes misfits between the experimental and theoretical data during EXAFS modelling. Amplitude reduction factor $\left(S_{0}{ }^{2}\right)$ was $0.67 . \sigma^{2}$ Indicates the 'Mean Squared Relative Displacement' between the absorber and scatterer.

\begin{tabular}{ccccccccc}
\hline $\begin{array}{c}\text { Charge } \\
\text { point }\end{array}$ & $\begin{array}{c}\text { Chemical } \\
\text { Potential }(\mathrm{V})\end{array}$ & R-range $(\AA)$ & $\mathrm{CN}$ & $\begin{array}{c}\mathbf{1}^{\text {st }} \text { shell } \\
\text { Co-O } \\
(\AA)\end{array}$ & $\begin{array}{c}\mathbf{2}^{\text {nd }} \text { shell } \\
\text { Co-TM } \\
(\AA)\end{array}$ & $\begin{array}{c}1^{\text {st }} \text { shell } \\
\left(\sigma^{2}\right)\end{array}$ & $\begin{array}{c}2^{\text {nd }} \text { shell } \\
\left(\sigma^{2}\right)\end{array}$ & R-factor \\
\hline REF. & 3.6098 & $1.1-3.65$ & 6 & 1.91119 & 2.80969 & 0.0029 & 0.01049 & 0.0147884 \\
1 & 4.326 & $1.15-3.6$ & 6 & 1.89191 & 2.79041 & 0.00177 & 0.01072 & 0.0206226 \\
2 & 4.5064 & $1-3.6$ & 6 & 1.89911 & 2.79761 & 0.00249 & 0.0004 & 0.0161627 \\
3 & 4.7507 & $1.05-3.6$ & 6 & 1.90137 & 2.79987 & 0.00242 & 0.01077 & 0.0196781 \\
4 & 3.8513 & $1.1-3.55$ & 6 & 1.90259 & 2.80109 & 0.00281 & 0.01219 & 0.0120153 \\
5 & 2.9265 & $1.1-3.65$ & 6 & 1.90772 & 2.80621 & 0.00243 & 0.00815 & 0.0118251 \\
6 & 2.1822 & $1.1-3.6$ & 6 & 1.90938 & 2.80788 & 0.00229 & 0.0078 & 0.0097386
\end{tabular}


Supplementary Table 3 | EXAFS fitting parameters at $\mathrm{Ni}$ K-edge on the graphene incorporated $\mathrm{Li}\left(\mathrm{Li}_{0.2} \mathrm{Mn}_{0.54} \mathrm{Ni}_{0.13} \mathrm{Co}_{0.13}\right) \mathrm{O}_{2}$. $\mathrm{CN}$ is the coordination number, $\mathrm{R}$-range is the fitting range, and $\mathrm{TM}$ is the transition metals ( $\mathrm{Mn}, \mathrm{Co}$, and $\mathrm{Ni}$ ). R-factor characterizes misfits between the experimental and theoretical data during EXAFS modelling. Amplitude reduction factor $\left(S_{0}{ }^{2}\right)$ was 0.92. $\sigma^{2}$ Indicates the 'Mean Squared Relative Displacement' between the absorber and scatterer.

\begin{tabular}{ccccccccc}
\hline $\begin{array}{c}\text { Charge } \\
\text { point }\end{array}$ & $\begin{array}{c}\text { Chemical } \\
\text { Potential } \\
(\mathrm{V})\end{array}$ & R-range $(\AA)$ & $\mathrm{CN}$ & $\begin{array}{c}\mathbf{1}^{\text {st }} \text { shell } \\
\text { Ni-O } \\
(\AA)\end{array}$ & $\begin{array}{c}1^{\text {st }} \text { shell } \\
\text { Ni-O } \\
\text { [short } \\
(\AA)\end{array}$ & $\begin{array}{c}1^{\text {st }} \text { shell } \\
\left(\sigma^{2}\right)\end{array}$ & $\begin{array}{c}2^{\text {nd }} \text { shell } \\
\left(\sigma^{2}\right)\end{array}$ & R-factor \\
\hline REF. & 3.6098 & $1.1-3.5$ & - & 2.03278 & - & 0.00464 & - & 0.0195 \\
1 & 4.326 & $1-3.55$ & $5.1,0.9$ & 2.04223 & 2.01392 & 0.00351 & 0.0079 & 0.0204 \\
2 & 4.5064 & $1.05-3.65$ & $4.6,1.4$ & 2.10324 & 2.00665 & $1.4 \mathrm{E}-4$ & 0.01404 & 0.0119 \\
3 & 4.7507 & $1.05-3.65$ & $4.6,1.4$ & 2.09144 & 1.99027 & 0.00322 & 0.00374 & 0.00966 \\
4 & 3.8513 & $1-3.6$ & 5,1 & 2.0413 & 2.01816 & 0.00364 & 0.0059 & 0.01073 \\
5 & 2.9265 & $1-3.55$ & 5,1 & 2.04217 & 2.02788 & 0.00433 & 0.00617 & 0.01442 \\
6 & 2.1822 & $1.1-3.55$ & - & 2.03853 & 2.01081 & 0.00709 & - & 0.02073
\end{tabular}

